\title{
Direct injection of fully stripped carbon ions into a fast-cycling induction synchrotron and their capture by the barrier bucket
}

\author{
N. Munemoto, ${ }^{1,2}$ S. Takano, ${ }^{2}$ E. Kadokura, ${ }^{2}$ Taufik, ${ }^{2,3}$ T. Yoshimoto ${ }^{4}$ X. Liu, ${ }^{5}$ T. Adachi, ${ }^{2,3}$ \\ M. Ikeda, ${ }^{2}$ T. Kawakubo, ${ }^{2}$ K. Okamura, ${ }^{2,3}$ K. Takayama, ${ }^{2,3}$ and M. Wake ${ }^{2}$ \\ ${ }^{1}$ Department of Energy Science, Tokyo Institute of Technology, Nagatsuda, Kanagawa 152-8550, Japan \\ ${ }^{2}$ Accelerator Laboratory, High Energy Accelerator Research Organization (KEK), \\ Tsukuba, Ibaraki 305-0801, Japan \\ ${ }^{3}$ The Graduate University for Advanced Studies, Hayama, Kanagawa 240-0193, Japan \\ ${ }^{4}$ Facility for Rare Isotope Beams (FRIB), Michigan State University, East Lansing, Michigan 48824, USA \\ ${ }^{5}$ RIKEN, Wako, Saitama 351-0198, Japan
}

(Received 29 March 2017; published 9 August 2017)

\begin{abstract}
Fully stripped carbon ions generated in a laser-ablation ion source are directly injected into a smallscale induction synchrotron. Carbon-ion beams of $\mathrm{C}^{6+}, \mathrm{C}^{5+}$, and $\mathrm{C}^{4+}$ are trapped in the barrier bucket. Beam properties, such as the momentum spread and beam lifetime in the low-energy ring, are measured and discussed in relation to the essential characteristics required in a carbon driver for future carbon therapies.
\end{abstract}

DOI: 10.1103/PhysRevAccelBeams.20.080101

\section{INTRODUCTION}

Energy sweep extraction in a single acceleration cycle from a fast-cycling synchrotron has been proposed [1], where a fast-cycling induction synchrotron requiring no large-scale injector is used as a driver and a beam is continuously extracted by a new beam-handling method to allow continuous beam-spill loss from the barrier bucket. A ring lattice with a large flat dispersion function has been designed for this technique. Extensive work to realize this scheme has been performed at KEK, Tsukuba, Japan since 2013, including an experimental demonstration of the fast-cycling induction synchrotron [2], an advanced beam-handling technique using the barrier bucket [3], and a laser-ablation ion source (LAIS) capable of generating fully stripped carbon ions $[4,5]$.

Recently, a fully stripped carbon-ion beam produced by the LAIS was directly injected into the fast-cycling induction synchrotron called the KEK Digital Accelerator (DA) and captured by the barrier bucket for its lifetime, which is determined by electron capture caused by the interaction with residual atoms and molecules. The direct injection in circular accelerator complexes, which has been never achieved so far, is the first step in creating a hadron driver for the next generation of cancer therapy.

Previously, $\mathrm{C}^{6+}$ injection from an ion source to an accelerator has been avoided because of concerns about

Published by the American Physical Society under the terms of the Creative Commons Attribution 4.0 International license. Further distribution of this work must maintain attribution to the author(s) and the published article's title, journal citation, and DOI. beam purity, although it is possible to generate $\mathrm{C}^{6+}$ in a high-frequency, high-power electron cyclotron resonance ion source (ECRIS). Even in the absence of air leaks, oxygen originating from the ion-chamber surface is a major source of contamination as $\mathrm{O}^{8+}$. In current carbon therapies, $\mathrm{C}^{4+}$ is usually produced from propane $\left(\mathrm{C}_{3} \mathrm{H}_{8}\right)$ or $\mathrm{CO}_{2}$ working gas in an ECRIS. $\mathrm{C}^{4+}$ ions are separated from the extracted carbon ions and injected into a radiofrequency quadrupole (RFQ) and linear accelerator, are converted to $\mathrm{C}^{6+}$ through the charge-exchange process in a stripper material, and then are injected into a slowcycling synchrotron to increase their energy to the required level.

Anani'n and colleagues demonstrated the acceleration of carbon ions formed by an LAIS in the Synchrophasotron at the Joint Institute for Nuclear Research, Dubna, Russia [6]. Carbon ions are accelerated in a preinjector and LU-20 linear accelerator, which provide an energy of $5 \mathrm{MeV} / \mathrm{u}$. The carbon ions are injected into the Synchrophasotron, accelerated to relativistic energies, and extracted with a carbon intensity on the order of $10^{5}$ particles per pulse. An LAIS and an accelerator system integrating the LAIS and a conventional accelerator have been investigated since then. Okamura and colleagues produced a $\mathrm{C}^{6+}$ beam of several tens of milliamps in their LAIS driven by a medium-energy $\mathrm{CO}_{2}$ laser (4 J) [7] and attempted immediate acceleration of a carbon-ion beam by an RFQ without charge separation. Based on these studies, research has begun on practical application of these carbon-ion beams in particle cancer therapy at various institutes, including in the private sector [8].

LAIS research was initiated in 2013 at KEK. A LAIS driven by a small Nd-YAG laser of $600 \mathrm{~mJ}$ with a graphite 


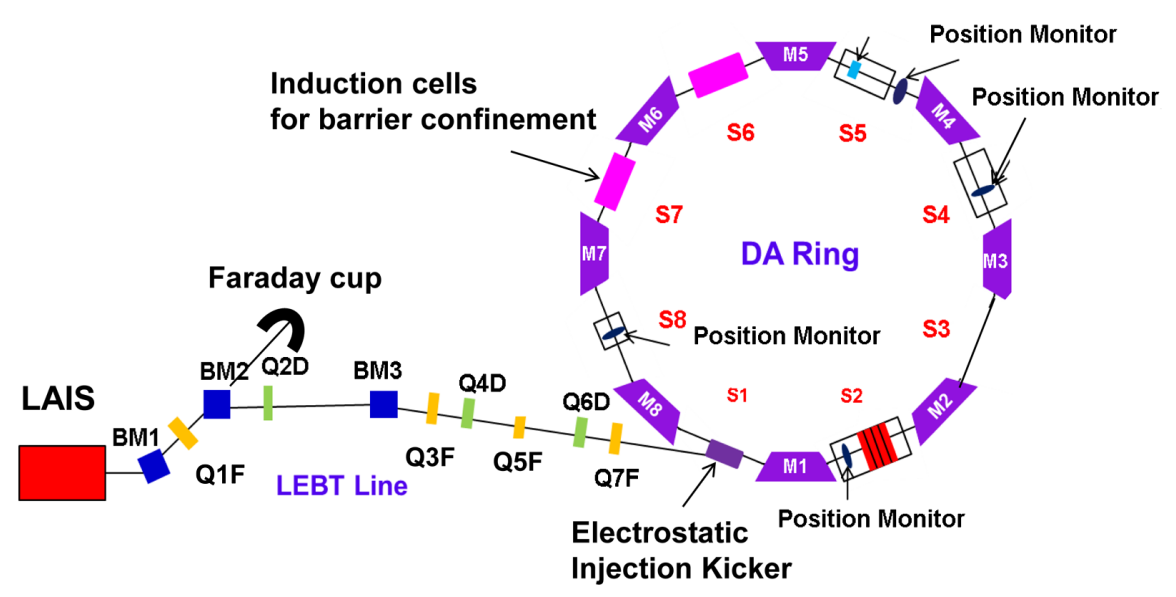

FIG. 1. Schematic view of the KEK-DA integrated with the LAIS.

target was completed in 2016 [4]. To simulate the key features of the future hadron driver experimentally, the LAIS was integrated with the KEK-DA, where a $\mathrm{C}^{6+}$ bunch is captured in the barrier bucket for its lifetime. In the near future, the captured $\mathrm{C}^{6+}$ bunch will be accelerated by the induction flat pulse voltage, as in any induction synchrotrons $[2,9]$. In this paper, we discuss how $\mathrm{C}^{6+}$ beams are obtained in the KEK-LAIS, transferred to the KEK-DA ring, and trapped in a barrier bucket. Some beam properties of the injected $\mathrm{C}^{4+}, \mathrm{C}^{5+}$, and $\mathrm{C}^{6+}$ beams are determined.

\section{ACCELERATOR SYSTEM}

\section{A. Outline}

The accelerator system integrating the LAIS and KEKDA is shown in Fig. 1. The laser irradiation chamber is embedded in the high-voltage platform (HVP) of the core body of the LAIS, which accommodated the ECRIS until 2015. Other accelerator components, such as the low-energy beam transport line (LEBT), electrostatic injection kicker, and DA ring, have the same setup as previously [2].

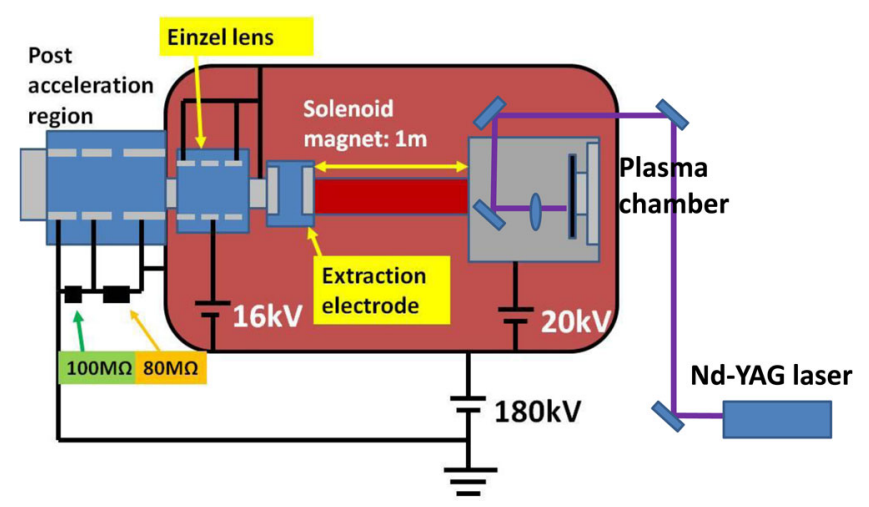

FIG. 2. The HVP for the LAIS system.

\section{B. LAIS and carbon ions}

The HVP and laser system for the LAIS are shown in Fig. 2. The HVP accommodates the LAIS irradiation chamber, guiding solenoid, extraction region, Einzel lens, postacceleration column, vacuum system, and power supplies. The KEK-LAIS chamber (Fig. 3) consists of the optical components and target unit. A Nd:YAG driving laser (SL800, Spectron; $0.6 \mathrm{~J} /$ pulse, $20 \mathrm{~ns}, 10 \mathrm{~Hz}$ ) is used. The laser beam is guided to the LAIS irradiation chamber with three $90^{\circ}$ mirrors. The laser path length to the target is about $5 \mathrm{~m}$. The repetition rate of the laser trigger is limited to $0.5 \mathrm{~Hz}$ because of the vacuum degradation in the LAIS chamber. The laser beam is focused by the focusing lens $(\phi=25 \mathrm{~mm}, f=100 \mathrm{~mm})$. These optical components are protected from the carbon vapor generated from the laser spot on the target surface by a thin metal shielding cover. The position of the focusing lens is adjusted to obtain as many $\mathrm{C}^{6+}$ ions as possible. A graphite target $(150 \times 150 \mathrm{~mm}$ and $1.7 \mathrm{~g} / \mathrm{cm}^{3}$ ) is mounted on the $X-Y$ flat moving stage. To obtain reproducible carbon plasmas, the irradiated target surface is refreshed shot by shot by moving the $X-Y$ stage with $0.5-\mathrm{mm}$ steps according to a program.

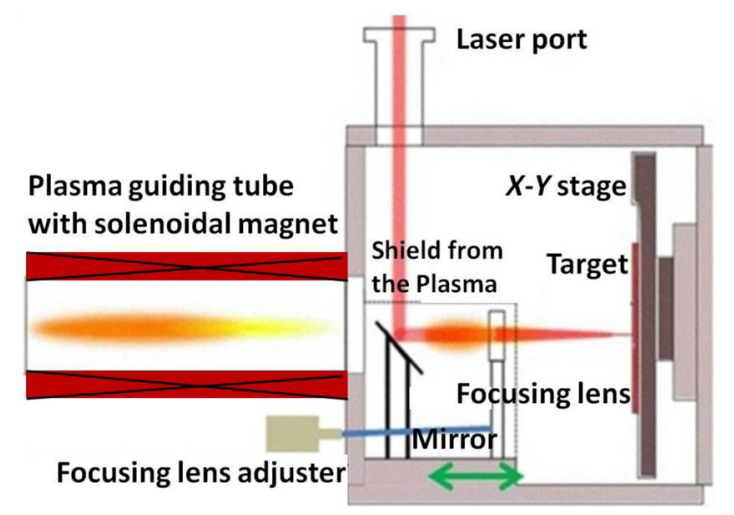

FIG. 3. The LAIS irradiation chamber. 


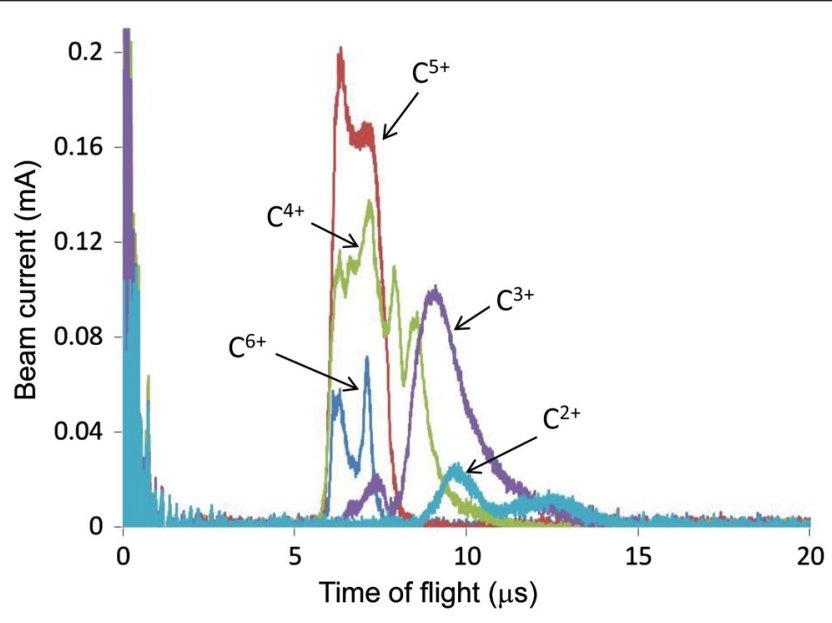

FIG. 4. Spectrum of carbon ions.

An ion beam is extracted from the LAIS through the extraction electrode. The distance from the target to the extraction gap is $1.3 \mathrm{~m}$. Over this distance, the carbon plasma is guided by the 1-m-long solenoid magnet with a flux density of $50 \mathrm{G}$. Planar extraction electrodes with an aperture of 2.8 and $4 \mathrm{~mm}$ in diameter and a gap distance of $18 \mathrm{~mm}$ are used for carbon-ion extraction [5]. A voltage of $20 \mathrm{kV}$ is applied across the extraction gap. The extracted beam is focused by an Einzel lens, to which an optimized voltage of $16 \mathrm{kV}$ is applied across the middle electrode. The extracted carbon beam is postaccelerated with a voltage of $200 \mathrm{kV}$. The plasma chamber and Einzel lens are under vacuums of $9 \times 10^{-5}$ and $3 \times 10^{-6} \mathrm{~Pa}$, respectively.

A charge-state spectrum observed by the Faraday cup, which is located downstream of the momentum-analyzer (or charge-separation) magnet BM1, is shown in Fig. 4. The extracted carbon ions are distributed from $\mathrm{C}^{2+}$ to $\mathrm{C}^{6+}$. The higher-charge-state carbon ions have a narrow momentum spread compared with the lower-charge-state carbon ions. This discrepancy is attributed to the laser ionization mechanism on the target surface [10]. In principle, the pulse length is determined by the initial momentum distribution created through the almost instantaneous laser-ablation ionization mechanism; faster ions gather in the bunch head and slower ions gather in the bunch tail. The number of particles (full width) and other beam parameters for individual charge states are given in Table I. This paper focuses on the high-charge-state carbon ions $\mathrm{C}^{6+}, \mathrm{C}^{5+}$, and $\mathrm{C}^{4+}$.

TABLE I. Parameters of carbon ions.

\begin{tabular}{lccc}
\hline \hline & $\mathrm{C}^{6+}$ & $\mathrm{C}^{5+}$ & $\mathrm{C}^{4+}$ \\
\hline Number of particles (one shot) [particles] & 0.56 & 3.37 & 4.77 \\
Peak current $[\mu \mathrm{A}]$ & 72 & 200 & 137 \\
Pulse width (FWHM) $[\mu \mathrm{s}]$ & 1.2 & 1.5 & 2.8 \\
\hline \hline
\end{tabular}

TABLE II. Beam and machine parameters.

\begin{tabular}{lccc}
\hline \hline & $\mathrm{C}^{6+}$ & $\mathrm{C}^{5+}$ & $\mathrm{C}^{4+}$ \\
\hline Mass to charge ratio $A / Q$ & 2 & 2.4 & 3 \\
Relativistic $\beta\left(\times 10^{-2}\right)$ & 1.46 & 1.33 & 1.19 \\
Revolution period $T_{0}[\mu \mathrm{s}]$ & 8.6 & 9.4 & 10.6 \\
Magnetic flux density of main & 279 & 303 & 339 \\
$\quad$ magnet [Gauss] & & & \\
Momentum slippage factor $|\eta|$ & 0.8107 & 0.8108 & 0.8108 \\
\hline \hline
\end{tabular}

\section{KEK-DA}

KEK-DA [11] is a fast-cycling induction synchrotron that was created from the original KEK $500-\mathrm{MeV}$ booster synchrotron for protons. KEK-DA has been used to accelerate various heavy ions with induction step-voltage pulses, and several advanced beam-handling techniques using the barrier bucket have been developed [3]. A charged heavy ion beam delivered from the high-voltage ion source is injected into KEK-DA by the electrostatic injection kicker [12]. The original thyratron is used as a kicker voltage shunt switch. Its turn-on speed is crucial for achieving nonperturbed injection, and this is replaced by a matrix array of static induction thyristors [13]. The injected ion bunch is trapped by a pair of barrier voltage pulses that are output in the induction cells. Two-turn induction cells, in which the magnetic core is wound with two bare coppercoil turns, are used [14]. The induction cells are energized by the switching power supply only for generating the barrier bucket at the same timing every turn, because acceleration is not carried out.

\section{CAPTURE BY THE BARRIER BUCKET}

\section{A. Free run}

After the carbon-ion beam is accelerated with a voltage of $200 \mathrm{kV}$ and charge-separated, a carbon-ion beam with a

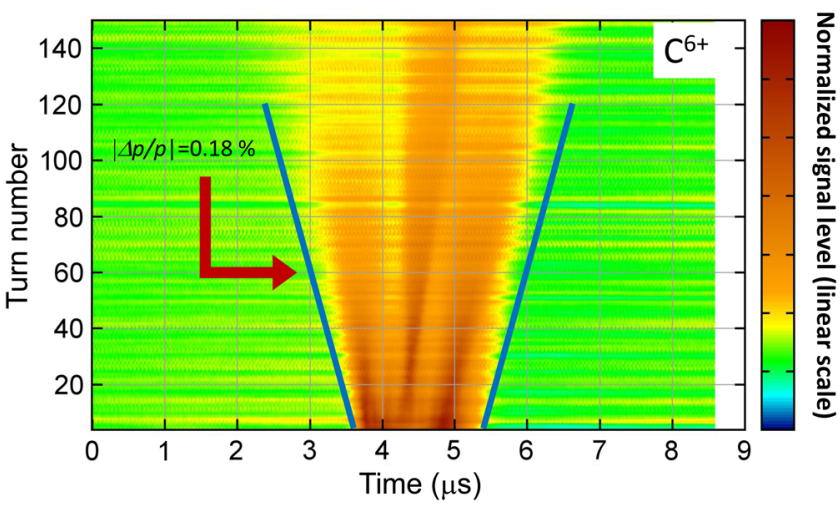

FIG. 5. Time-turn plane view of the free run of $\mathrm{C}^{6+}$, where some fine structures are visible. This fine structure in time of the beam pulse profile is known to be attributed to effects of the residual voltage ringing of the ES injection kicker [13]. 


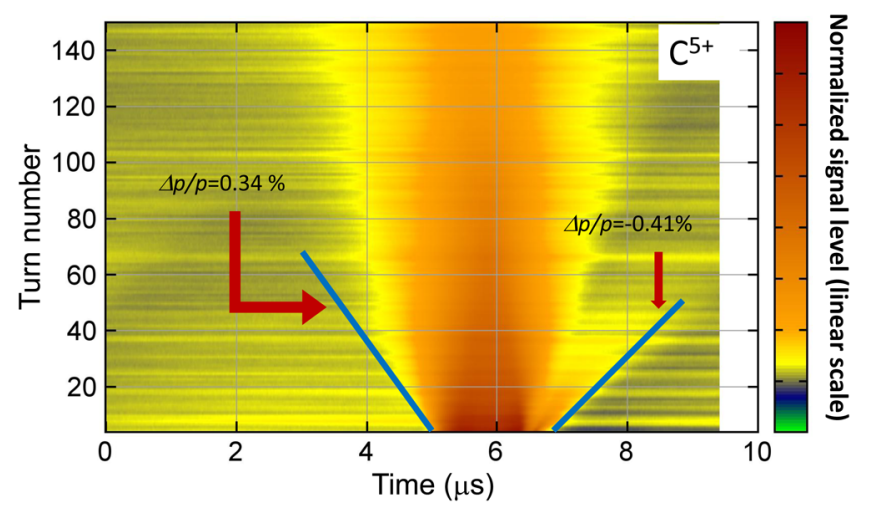

FIG. 6. Time-turn plane view of the free run of $\mathrm{C}^{5+}$ where a low-energy component in the tail is visible (color changed to make the image clear).

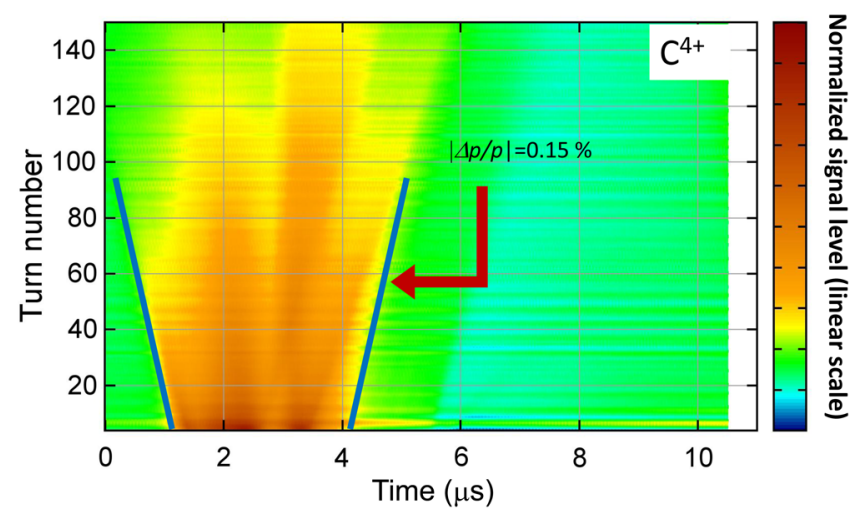

FIG. 7. Time-turn plane view of the free run of $\mathrm{C}^{4+}$.
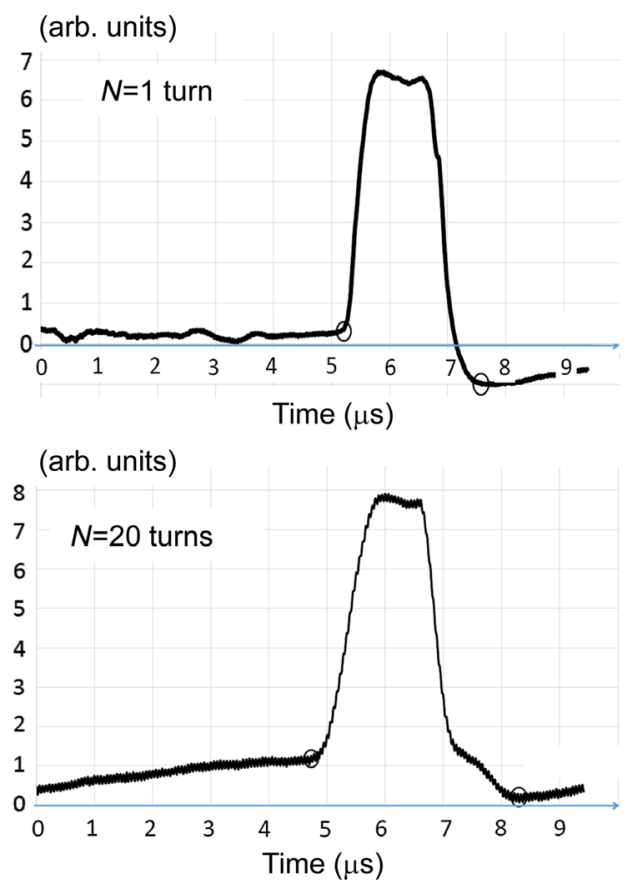

specific charge state is guided through the LEBT and injected into the KEK-DA ring, and the main magnet is excited at the fixed static magnetic field depending on the charge state of the injected carbon ion (Table II). The circulating beam in the ring is observed by the electrostatic position monitors placed at S4 and S8 (Fig. 1) that also function as current monitors.

Figures 5-7 show the profiles of the injected ion beam current, in which the ion beam current signal is projected on the time-turn plane with intensifying color imaging. The horizontal axis is the time corresponding to the revolution period $\left(\mathrm{C}^{6+}, 8.6 \mu \mathrm{s} ; \mathrm{C}^{5+}, 9.4 \mu \mathrm{s}\right.$; and $\mathrm{C}^{4+}$, $10.6 \mu \mathrm{s}$ ), and the vertical axis corresponds to the turn number. The color scale represents linearly the current density. The pulse widths of $\mathrm{C}^{6+}, \mathrm{C}^{5+}$, and $\mathrm{C}^{4+}$ are 2,2 and 3.0 $\mu \mathrm{s}$, respectively. These pulse widths are consistent with the results measured by the Faraday cup on the LEBT. The maximum momentum spread can be directly evaluated from the pulse profile on the time axis that evolves by $t_{n+1}=t_{n}+T_{0} \eta \Delta p / p$, where $n$ is the turn number, $t$ is the time, $T_{0}$ is the revolution time, and $\eta$ is the phase slippage factor. Actually, the inflection points of the line-density profile as shown in Fig. 8 that correspond to the outer edges of the beam pulse have been read. This procedure gives $\left|\Delta p / p_{\max }\right|=0.18 \%$ for $\mathrm{C}^{6+}, 0.41 \%<$ $\Delta p / p_{\max }<+0.34 \%$ for $\mathrm{C}^{5+}$, and $\left|\Delta p / p_{\max }\right|=0.15 \%$ for $\mathrm{C}^{4+}$. The momentum spread for $\mathrm{C}^{5+}$ is notably larger than those for $\mathrm{C}^{6+}$ and $\mathrm{C}^{4+}$. The reason for this is not known, although it may suggest higher longitudinal space-charge affects during the LEBT propagation. For the reference,
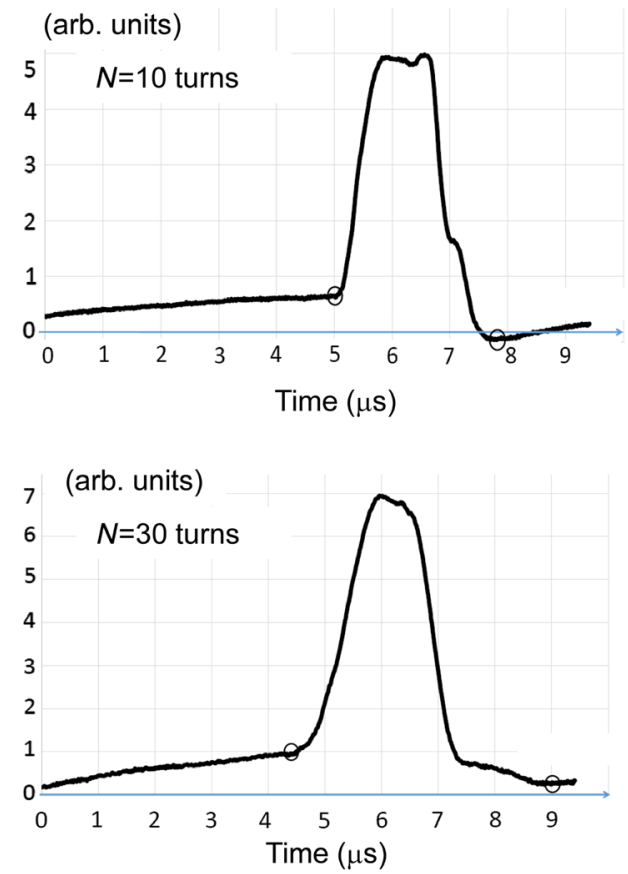

FIG. 8. Beam pulse signals for $\mathrm{C}^{5+}$ obtained by the electrostatic monitor. Circles denote the inflection points corresponding to both edges of a beam pulse. 


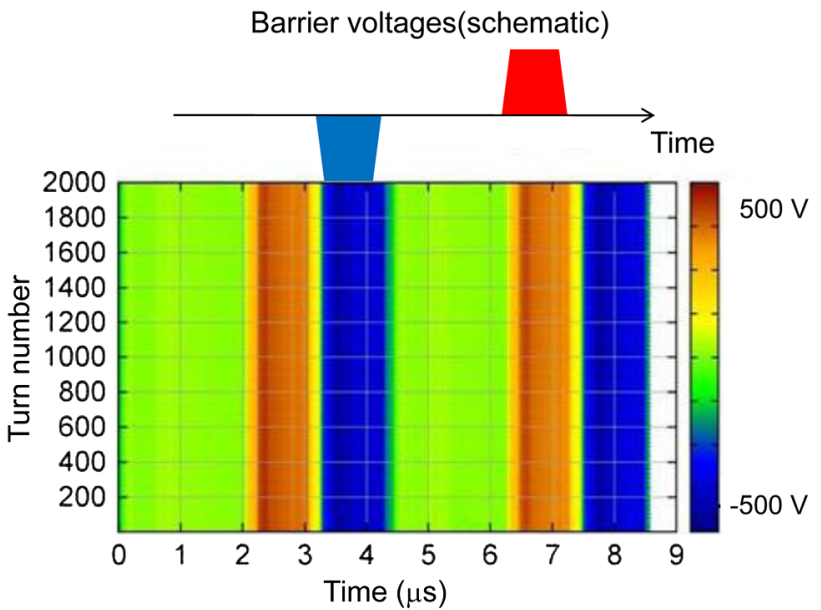

FIG. 9. Barrier voltage pulses used for $\mathrm{C}^{6+}$ confinement.

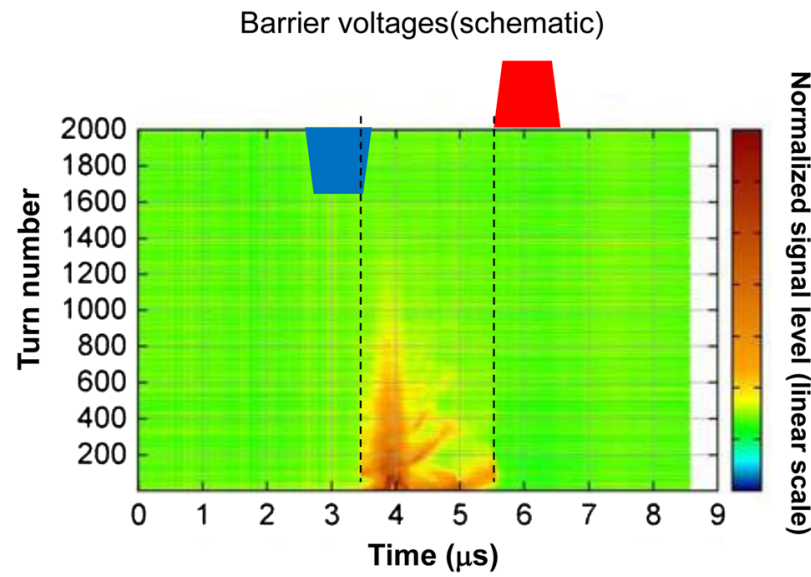

FIG. 10. Time-turn plane view of the trapped $\mathrm{C}^{6+}$ beam.

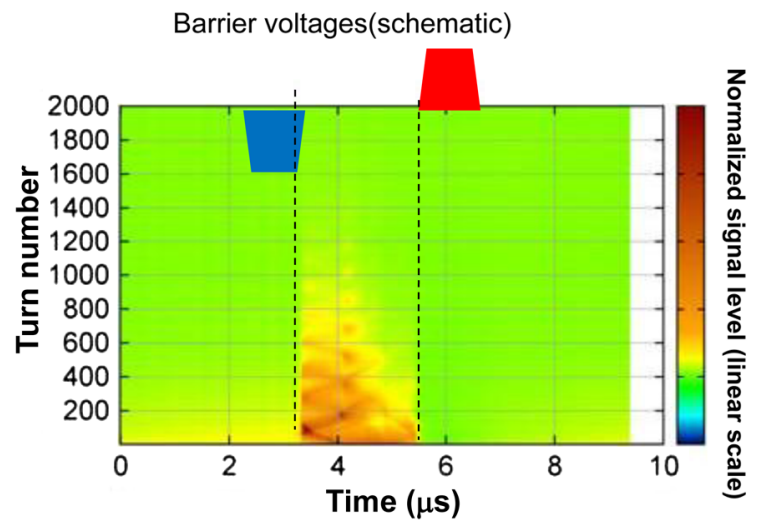

FIG. 11. Time-turn plane view of the trapped $\mathrm{C}^{5+}$ beam.

the evaluated diffusion lines are put on the above projected pulse profiles. One finds that the diffusion seen on the time-turn plane well follows the numerical evaluation.

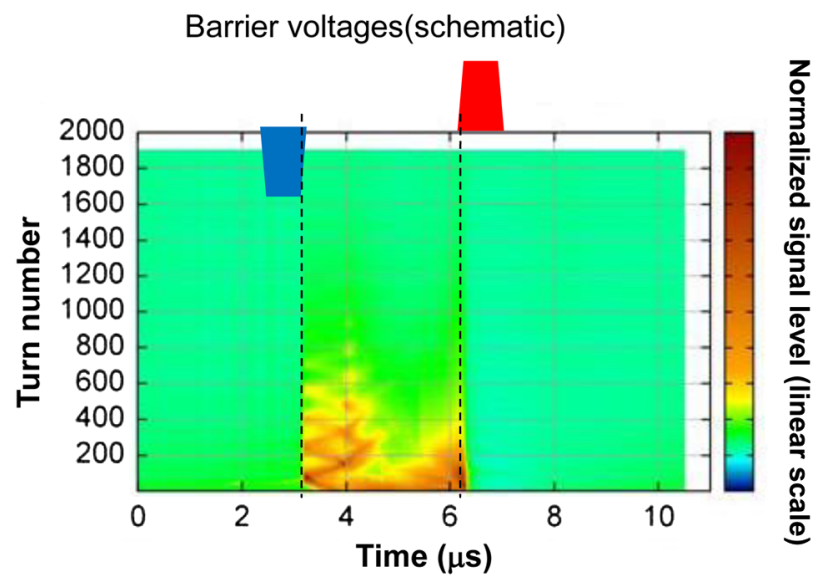

FIG. 12. Time-turn plane view of the trapped $\mathrm{C}^{4+}$ beam.
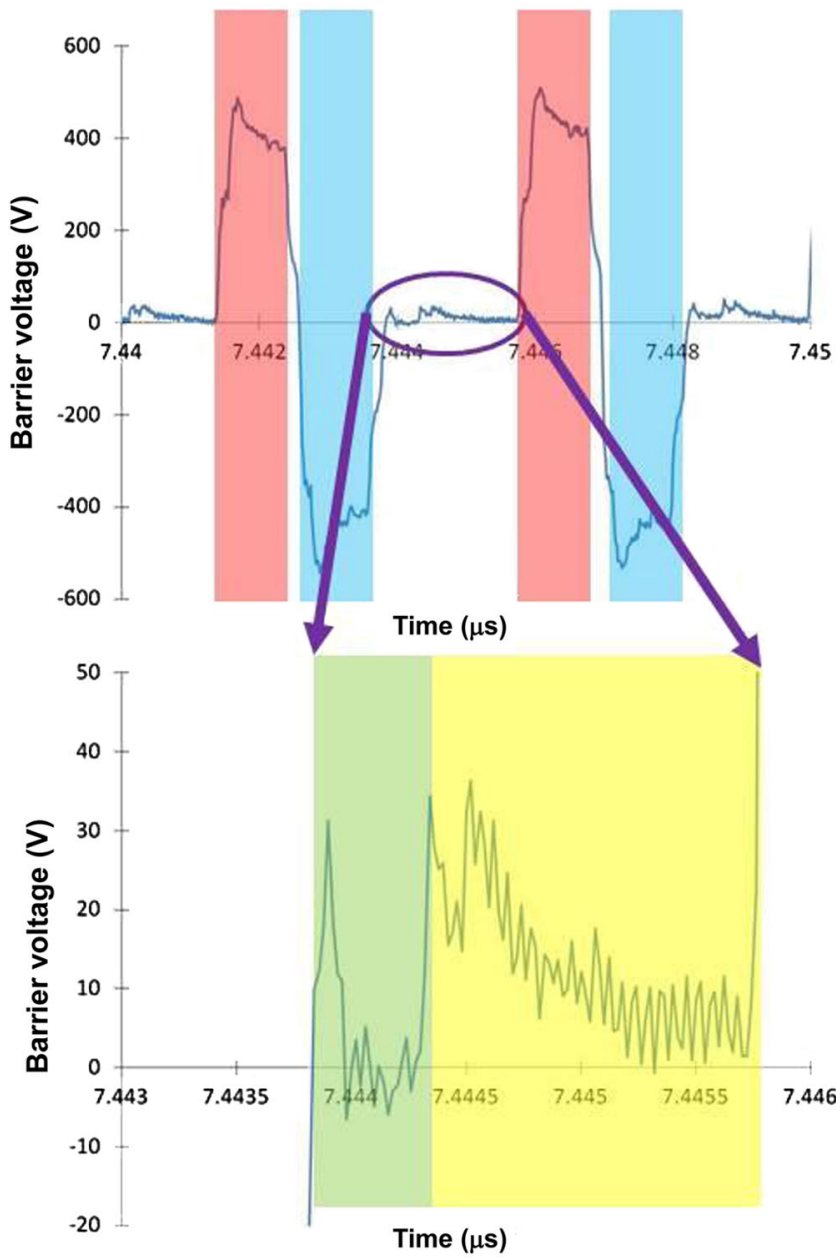

FIG. 13. Barrier voltage pulse profile with fine structure.

\section{B. Barrier capture}

In the induction synchrotron [15], longitudinal confinement is separated from acceleration. Two barrier voltage pulses are generated to prevent the beam from spreading in the longitudinal direction. The induction cells used for the 

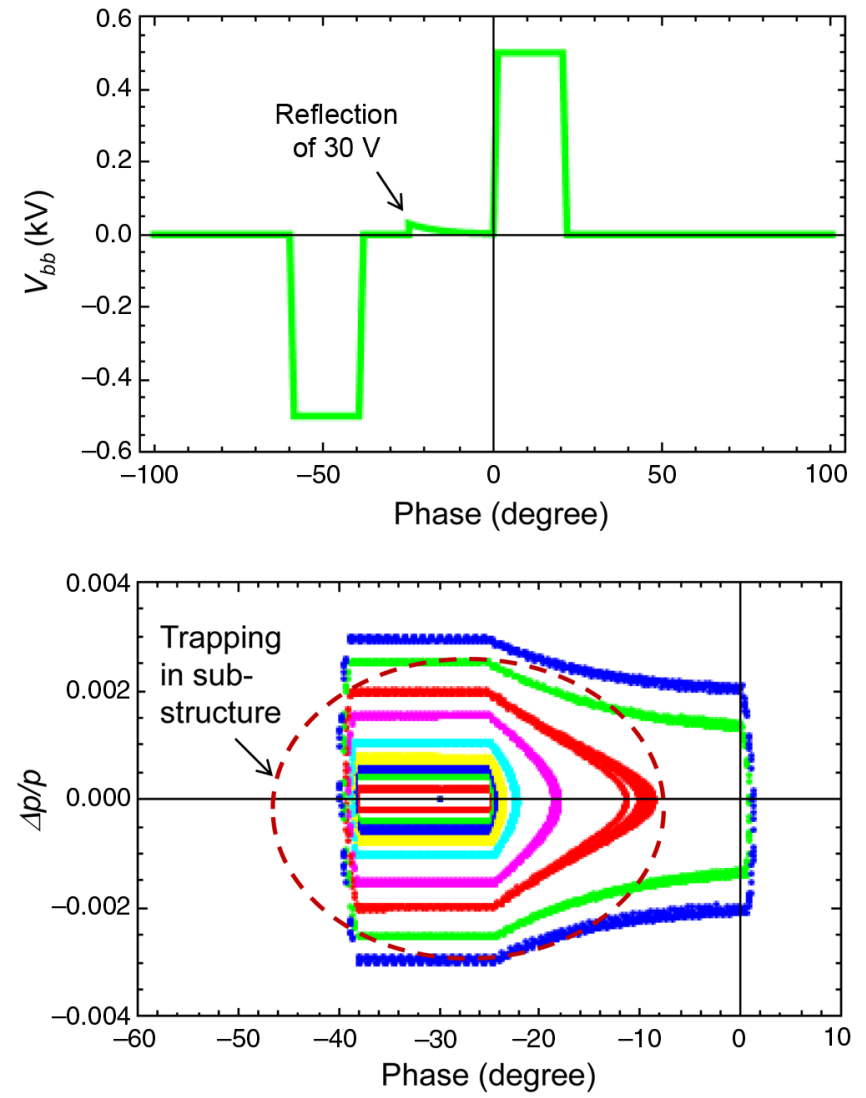

FIG. 14. (Upper) Model barrier voltage pulse with a reflection and (bottom) phase-space struture of the barrier bucket disturbed by the reflection voltage. confinement are located at S7 (Fig. 1). The time interval between the two pulses is kept the same as the beam length at injection. Figure 9 shows the barrier voltage of $\mathrm{C}^{6+}$ in the time-turn plane, where the voltage pulses are projected. The color scale reflects the magnitude of the barrier voltage. For these experiments, the maximum barrier voltages are fixed at $\pm 500 \mathrm{~V}$, and their pulse widths are $2 \mu \mathrm{s}$. The confinement time is $2 \mu$ s for $\mathrm{C}^{6+}$ and $\mathrm{C}^{5+}$ and $3.5 \mu$ s for $\mathrm{C}^{4+}$. The temporal evolution of the beam confinement in the timeturn plane is shown in Figs. 10-12, and it confirms that the beam signals remain for far longer than that of the free run described in Sec. III. A.

At first glance, the confined beam seems to curiously behave on the time-turn plane. The trapped beam is broadly confined in the created confinement area between the barrier voltage pulses; meanwhile, a fraction of the trapped bunch reflects at far front of the right barrier voltage pulse. We have observed this phenomenon in a previous iontrapping experiment using the KEK-DA [16]. It is known that the fraction of the trapped ions with a small momentum spread is trapped by a localized resonance island in the phase space, which is generated by the fine structure in the barrier voltage pulse profile. The fine structure in the barrier voltage profile is identified as a positive voltage bump of $35 \mathrm{~V}$ just after the negative barrier voltage pulse (Fig. 13).

In order to confirm this speculation, the phasespace structure, which is determined with the more realistic barrier voltage taking the refection into account,
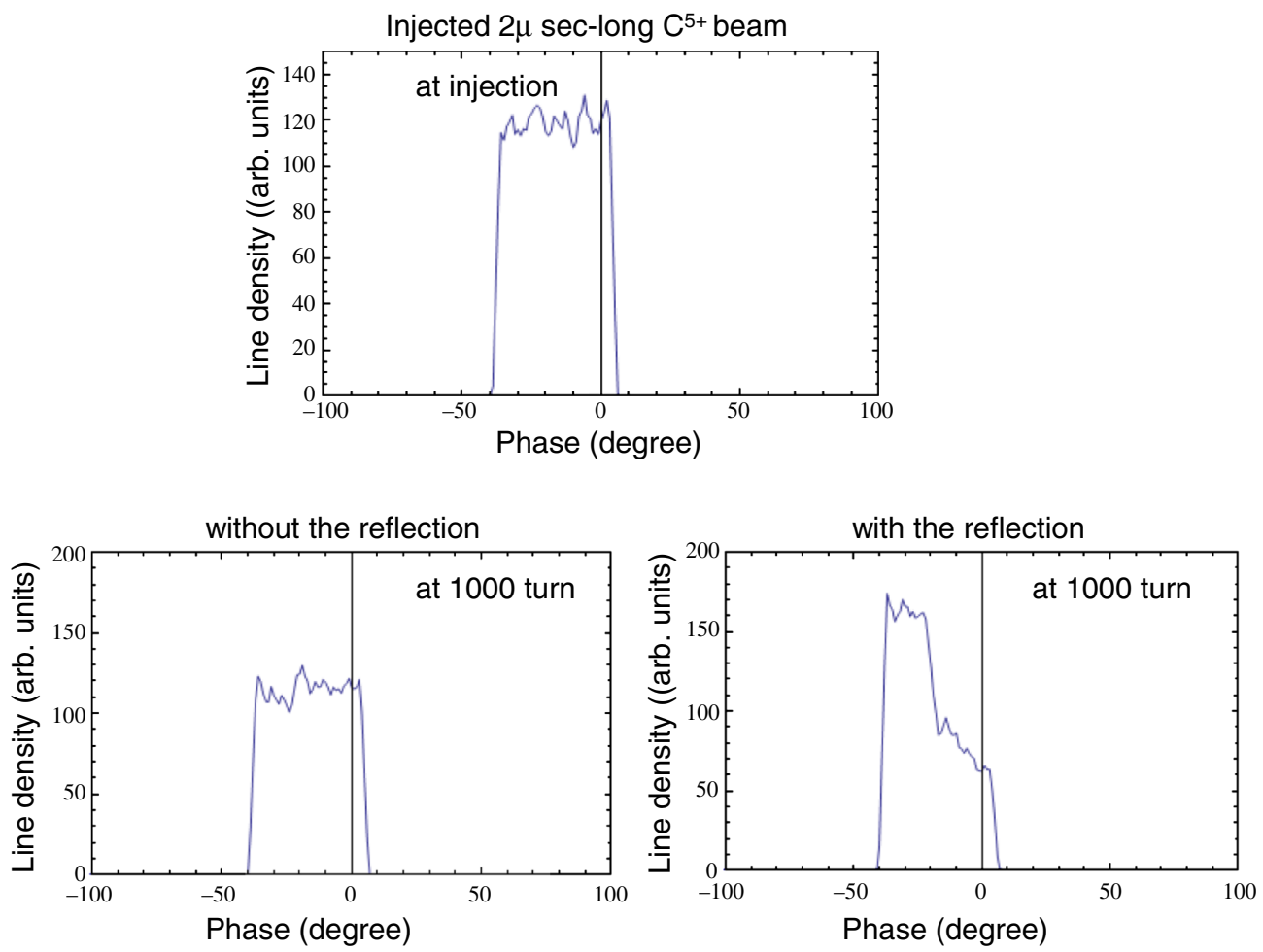

FIG. 15. Multiparticle-tracking simulation from a $\mathrm{C}^{5+}$ beam, (upper) the line density of an injected $\mathrm{C}^{5+}$ beam pulse uniformly distributed in the longitudinal direction, and (lower) line density after 1000 turns. 
is investigated. The substructure is obvious in Fig. 14, as predicted. Some ions of $\mathrm{C}^{5+}$ that are injected near the left barrier voltage pulse timing with a lower-momentum deviation are captured in this substruture. The positive reflection voltage takes a role of barrier there. This is a beam-dynamical reason why the line density relatively increases in the side of the left barrier. In addition, multiparticle-tracking simulations are carried out, although the precise distribution in the momentum space except for the maximum deviation is not known. The result showing the line density at 1000 turns seems to reproduce qualitatively the experimental result (Fig. 15).

\section{BEAM LIFETIME}

The temporal evolution of the beam intensity signal indicates the net beam lifetime. The intensity of a carbonion beam trapped in the barrier bucket should be conserved without external perturbations. However, there are many perturbation sources that determine the lifetime.

The following sources lead to unavoidable steady-state loss: (i) electron capture as a result of interactions with residual atoms and molecules and (ii) electron stripping (for $\mathrm{C}^{5+}$ or below) as a result of interactions with residual atoms and molecules.

The cross sections for these interactions, which are a function of the projectile ion velocity, have been reported for limited atom species [17]. In the current injection energy, source (i) is dominant and source (ii) does not apply to $\mathrm{C}^{6+}$. The beam lifetime due to source (i) is described by

$$
N(t)=N(0) \exp \left[-2.12 \times 10^{27} P(\text { torr }) \int_{0}^{t}\left(\sigma_{\text {cap }} \beta\right) d t^{\prime}\right],
$$

where $N(0)$ is the initial number of ions, $t$ is the time from injection, $\beta$ is the relativistic beta, $P$ is the pressure in Torr, and $\sigma_{\text {cap }}$ is the electron-capture cross section. This equation is more simplified and written as a function of turn number $n$, because ions are not accelerated:

$$
\begin{aligned}
N(n) & =N(0) \exp \left[-2.12 \times 10^{27} P(\text { torr }) \sigma_{\text {cap }} \beta T_{0} n\right] \\
& =n(0) \exp \left(-\frac{n}{n_{\text {life }}}\right)
\end{aligned}
$$

with

$$
n_{\text {life }}=\frac{1}{2.12 \times 10^{27} \beta T_{0} P(\text { torr }) \sigma_{\text {cap }}},
$$

where $T_{0}$ is the revolution time period.

With the substitution of $T_{0}=9.4 \mu \mathrm{s}, \beta=1.33 \times 10^{-2}$, and observed averaged pressure $P=1.5 \times 10^{-8}$ into Eq. (2), we have $n_{\text {life }}=2.51 \times 10^{-13} / \sigma_{\text {cap }}$.

We do not measure the contents of the residual atoms and molecules by using a specific tool such as Q-MASS. There is no clear vacuum leak. The assumption that molecules

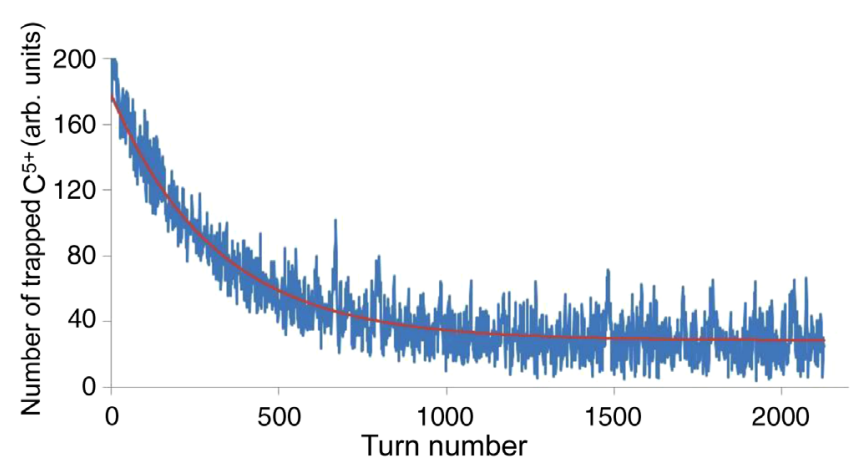

FIG. 16. Beam survival of $\mathrm{C}^{5+}$.

and atoms originate from $\mathrm{H}_{2} \mathrm{O}$ emitted from the chamber surface appears to be correct, similar to other hadron synchrotrons operated under a moderate vacuum. Whatever the residual molecules are, it seems to be reasonable to assume that the cross sections of hydrogen, helium, and nitrogen molecule are almost similar for the current projectile ion velocity, as shown in the literature [17], although the data of the oxygen molecule are not available there. Substituting $\sigma_{\text {cap }}=8 \times 10^{-16} \mathrm{~cm}^{2}$ into Eq. (2) and plotting the predicted beam intensity function $n(t)$ over the experimental data, we find a good agreement in Fig. 16. The experimental data for $\mathrm{C}^{5+}$ are used, because the electron capture cross section for $\mathrm{C}^{6+}$ is unknown.

In addition to electron capture resulting in substantial beam loss, the following sources cause emittance blowup and result in loss: (i) coherent beam centroid oscillation and envelope oscillation associated with injection errors, (ii) nonlinear resonance crossing caused by space-charge forces, (iii) Coulomb scattering with residual charged atoms and molecules, (iv) intrabeam scattering, and (v) chaotic motion due to barrier voltage confinement, resulting in longitudinal emittance blowup (loss from the barrier bucket) [18].

Sources (i) and (ii) are responsible for fast loss observed just after injection and are beyond the scope of the present discussion. Source (iii) depends directly on pressure. Source (iv) depends on the beam emittance and intensity. Source (v) is determined by the barrier voltage pulse shape, especially the voltage rise time. A rough estimation using the known formula for these processes suggests that emittance blowup is slow and not important in the current setup.

\section{DISCUSSION: SUMMARY AND FUTURE PROSPECTS}

A fully stripped carbon-ion beam delivered from the LAIS and directly injected into the KEK-DA is captured by the barrier bucket, which is expected to be a crucial beamhandling tool for a hadron driver in future cancer therapy [1]. The beam intensity of $\mathrm{C}^{6+}$ in the current LAIS is still insufficient; however, the momentum spread is extremely small and on the order of $10^{-3}$, retaining the original momentum spread generated in the LAIS. The beam 
intensity is determined by the laser power density on the target spot. An optimized, more powerful laser will be required to provide $\mathrm{C}^{6+}$ with $10^{8} /$ pulse. However, a laser that is too powerful increases debris, including lowercharge-state carbon ions that create a load on the vacuum in the irradiation chamber, resulting in plasma loading on the high-voltage power supply [19] and greatly reducing the voltage. The current repetition rate of the LAIS is limited to $0.5 \mathrm{~Hz}$ because of the degradation of the vacuum level in the irradiation chamber. Upgrading the capability of the evacuation system is expected to increase the repetition rate.

The present barrier bucket capture experiment suggests the necessary vacuum level in the driver ring. Although quick acceleration can largely mitigate electron capture by $\mathrm{C}^{6+}$, a vacuum of $10^{-7} \mathrm{~Pa}$ is required.

A better vacuum is planned for the KEK-DA, where all outgassing accelerator components responsible for degrading the vacuum, such as the extraction kickers, will be removed from the beam line. Furthermore, the induction acceleration system will be improved by introducing a variable dc power supply, without which the KEK-DA has had to accelerate heavy ions discretely with pulse-density control [14], inducing beam centroid oscillation with a large amplitude due to synchrobeta coupling, which results in emittance blowup [2].

\section{ACKNOWLEDGMENTS}

The collaboration with Dr. M. Okamura (BNL) and his team initiated the current KEK-LAIS project. We thank Dr. Y. Iwata (AIST) for providing valuable suggestions and the current laser system, without which we would not have generated $\mathrm{C}^{6+}$ ions. N. M. thanks Professor Horioka (TIT) for continuous and helpful advice. The initial stage of this study was financially supported by Grants-In-Aid for Scientific Research (A) (KAKENHI No. 23240082).

[1] L. K. Wah, T. Monma, T. Adachi, T. Kawakubo, T. Dixit, and K. Takayama, A compact hadron driver for cancer therapies using continuous energy sweep scanning, Phys. Rev. Accel. Beams 19, 042802 (2016).

[2] K. Takayama T. Yoshimoto, M. Barata, L. K. Wah, L. Xingguang, T. Iwashita, S. Harada, T. Adachi, T. Arai, D. Arakawa, H. Asao, E. Kadokura, T. Kawakubo, H. Nakanishi, Y. Okada, K. Okamura, K. Okazaki, A. Takagi, S. Takano, and M. Wake, Induction acceleration of heavy ions in the KEK digital accelerator: Demonstration of a fast cycling induction synchrotron, Phys. Rev. ST Accel. Beams 17, 010101 (2014).

[3] T. Yoshimoto, Doctor thesis, Tokyo Institute of Technology, 2016. Historically, the barrier bucket was demonstrated as an isolated rf bucket by Griffin et al. at Fermilab in 1983. There are big differences between the isolated rf barrier bucket and induction barrier bucket. Their details are discussed in the textbook Induction Accelerators, edited by K. Takayama and R. J. Briggs (Springer, Heidelberg, 2011). Useful references describing the evolution of rf barrier bucket technology thereafter are therein.

[4] N. Munemoto, K. Takayama, S. Takano, M. Okamura, and M. Kumaki, Development of the $\mathrm{C}^{6+}$ laser ablation ion source for the KEK digital accelerator, Rev. Sci. Instrum. 85, 02B922 (2014).

[5] N. Munemoto, S. Takano, K. Takayama, K. Okamura, and K. Horioka, in Proceedings of IPAC2016 (unpublished), WEPMY036.

[6] O. B. Anan'in et al., Realization of acceleration of laserinjector-generated carbon nuclei in the Synchrophasotron at the Joint Institute for Nuclear Research, Sov. J. Quantum Electron. 7, 873 (1977).

[7] H. Kashiwagi, M. Fukuda, M. Okamura, R. A. Jameson, T. Hattori, N. Hayashizaki, K. Sakakibara, J. Takano, K. Yamamoto, Y. Iwata, and T. Fujimoto, Acceleration of high current fully stripped carbon ion beam by direct injection scheme, Rev. Sci. Instrum. 77, 03 B305 (2006).

[8] T. Sako, A. Yamaguchi, K. Sato, A. Goto, T. Iwai, T. Nayuki, K. Nemoto, T. Katayama, and T. Takeuchi, Development of $\mathrm{C}^{6+}$ laser ion source and RFQ linac for carbon ion radiotherapy, Rev. Sci. Instrum. 87, 02 C109 (2016).

[9] K. Takayama, Y. Arakida, T. Dixit, T. Iwashita, T. Kono, E. Nakamura, K. Otsuka, Y. Shimosaki, K. Torikai, and M. Wake, Experimental Demonstration of the Induction Synchrotron, Phys. Rev. Lett. 98, 054801 (2007).

[10] I. V. Roudskoy, General features of highly charged ion generation in laser-produced plasmas, Laser Part. Beams 14, 369 (1996).

[11] T. Iwashita et al., KEK digital accelerator, Phys. Rev. ST Accel. Beams 14, 071301 (2011).

[12] T. Adachi and T. Kawakubo, Electrostatic injection kicker for the KEK digital accelerator, Phys. Rev. ST Accel. Beams 16, 053501 (2013).

[13] H. Kobayashi, L. Xinggunag, T. Yoshimoto, K. Takayama, T. Kawakubo, and T. Adachi, Beam dynamics analysis of heavy ion injection into the KEK digital accelerator, Plasma Fusion Res. 11, 1404092 (2016).

[14] T. Dixit, T. Iwashita, and K. Takayama, Induction acceleration scenario from an extremely low energy in the KEK all-ion accelerator, Nucl. Instrum. Methods Phys. Res., Sect. A 602, 326 (2009).

[15] K. Takayama and J. Kishiro, Induction synchrotron, Nucl. Instrum. Methods Phys. Res., Sect. A 451, 304 (2000).

[16] M. Hirose, K. Takayama, T. Yoshimoto, and X. Liu, in Proceedings of HIAT2015 (unpublished), p. MOPA22.

[17] I. S. Dmitiev, Y. A. Teplova, Y. A. Belkova, N. V. Novikov, and Y. A. Fainberg, Experimental electron loss and capture cross sections in ion- atom collisions, At. Data Nucl. Data Tables 96, 85 (2010).

[18] K. Takayama, Y. Shimosaki, and M. Hirose, Barrier bucket confinement dynamics (to be published).

[19] K. Horioka (private communication). 